\title{
Simple technique for reconstruction of a bifuraction lesions of diffuse coronary artery disease
}

\author{
Sayed $\mathrm{S}^{1,2 *}$, Alassal MA ${ }^{3}$, Elrakhawy $\mathrm{HM}^{3}$ and Ibrahim $\mathrm{MF}^{4}$ \\ ${ }^{1}$ Klinik für Thorax-Herz und Thoracale Gefäßchirurgie, Universitätsklinikum Würzburg, Germany \\ ${ }^{2}$ Cardiothoarcic Surgery Department- Assiut University, Egypt \\ ${ }^{3}$ Cardiothoracic Surgery Department- Banha University, Egypt \\ ${ }^{4}$ Department of Cardiac surgery, Prince Salman Heart Center, King Fahad Medical City, Riyadh-Kingdom of Saudia Arbia
}

\begin{abstract}
We report a technique by which a bifurcation lesion of the first diagonal branch of the left anterior descending coronary artery can be bypassed after endarterectomy. After opening the artery proximally, the incision was extended to involve both limbs of the bifurcation. After complete endarterectomy inverted Y shaped anastomosis was designed using single vein graft. The technique is simple, reproducible and easily performed especially in small sized vessels after endartectomy. With this technique it is possible to perform 2 distal anastomoses using a single conduit without adding extra bypass time.
\end{abstract}

\section{Introduction}

Increasing numbers of patients with advanced coronary artery disease and diffusely complex atherosclerotic lesions are more often referred now for coronary artery bypass surgery [1]. Under these circumstances, complete revascularization with an adequate distal run off can only be achieved by extensive endarterectomy and reconstructive procedures [2].

Because of the numerous septal and diagonal branches of the left anterior descending coronary artery (LAD) and its great importance as a target graft in myocardial revascularization, grafting of a major diagonal branch is sometimes required for complete revascularization.

Different means of LAD reconstruction using endarterectomy with either long segmental anastomosis and or on-lay patches have been used with encouraging results [3]. However, bifurcation lesions represent some technical difficulties when performing anastomosis specially, if the lesion is long and complex.

We describe a technique of anastomosis by which a bifurcation lesion of any major coronary artery can be anastomosed to a vein graft after endarterectomy.

\section{Description of surgical technique}

After standard median sternotomy, LIMA and saphenous vein were harvested. The saphenous vein graft selected for this bypass must be of a good quality vessel with a diameter not less than $4 \mathrm{~mm}$ (preferably the thigh vein). Cardiopulmonary bypass was established by cannulating the distal ascending aorta and the right atrium, cold antegrade blood cardioplegia was infused through an aortic root cannula following aortic cross clamp and repeated every 20 minutes.

The target vessels were inspected, palpated and the site of the anastomosis was determined. On exploration of the $1^{\text {st }}$ diagonal branch of the left anterior descending artery, it was found that it is a big artery which bifurcates into two good sized vessels. There was a significant lesion at the bifurcation site including both vessels. A small incision was performed in the proximal part of the vessel just before bifurcation and extended to both sides of the bifurcation. An atheramotus plaque was found involving both limbs of the bifurcation. After meticulous endarterectomy the size of the incision was $3 \mathrm{~cm}$ long on both limbs of the bifurcation.

Vein was incised in the middle to have 2 equal limbs like a pantaloon. Anastomosis was performed with single suture $7 / 0$ polyprolene. Suturing technique begins just before bifurcation at 4 oclock position of the vein and 2' o'clock position of the artery moving in anti -clockwise direction (Figure 1)

After completion of the planned grafts and before weaning of the cardiopulmonary bypass, flow meter was performed to study the flow across the graft. It was $41 \mathrm{ml} / \mathrm{min}$, with pulsatliy index 2.5 (Veri Q Machine - Taiwan).

Coronary angiography was performed before discharge of the patient (Figure 2), which showed a reasonable filling at the site of the anastomosis. The patient had uneventful recovery and was discharged on the $7^{\text {th }}$ postoperative day

\section{Discussion}

The clinical profiles of patients referred now for coronary surgery are more complex. Most of the patients are referred with diffuse disease [4].

Bypass grafting of the diffusely diseased vessels usually require some complex surgical procedures like on-lay patches, endarterectomies with

${ }^{\star}$ Correspondence to: Sameh Sayed, Klinik für Herz Thorax und Thoracale Gefäßchirurgie, Universitätsklinikum Würzburg, Oberdürrbacher, WürzburgGermany, Tel: +4993120133021; Fax: +4993120133009; E-mail: samsayed06@gmail.com

Key words: reconstruction, bifurcation lesion, coronary artery disease

Received: June 04, 2018; Accepted: June 20, 2018; Published: June 26, 2018 


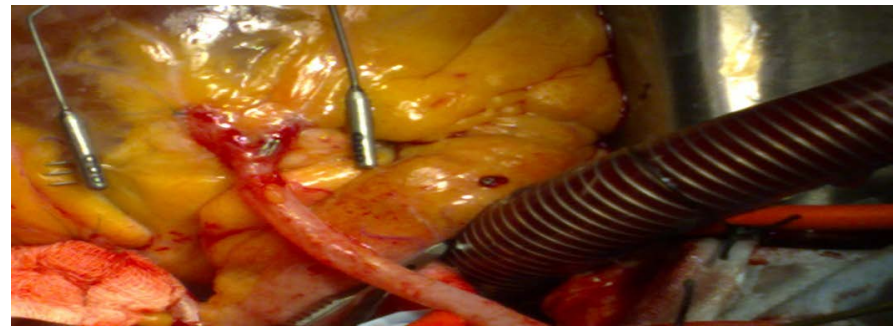

Figure 1. Intra-operative photo of our inverted Y graft to the first diagonal artery

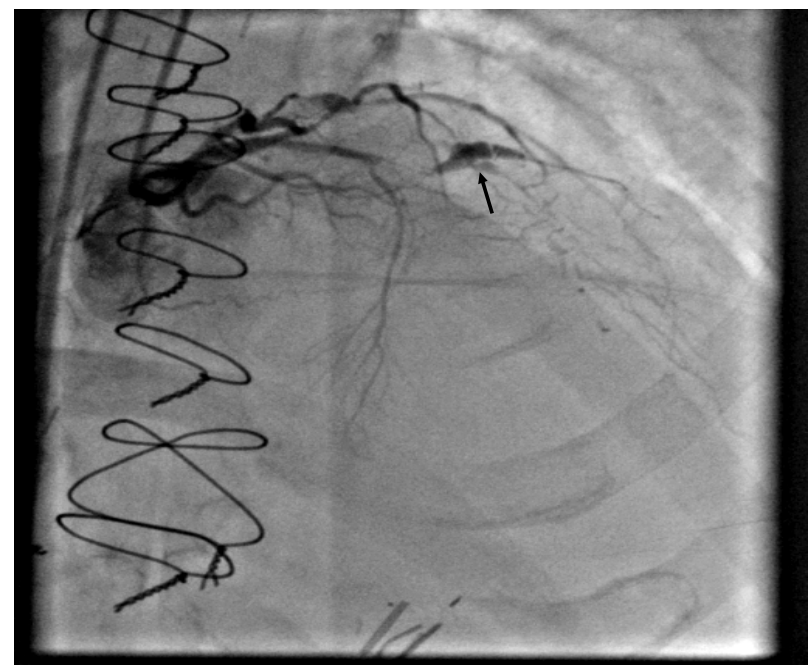

Figure 2. Postoperative Coronary Angiography showing a reasonable filling (Arrow) at the site of the anastomosis

longer anastomosis and sometimes more than one graft for the same vessel, however many surgeons are still reluctant to use these techniques because of its high perioperative and postoperative mortality rates [5].

Coronary endarterectomy is usually the technique of choice in patients presenting with diffuse and extensive coronary artery disease [6]. However, modifications of conventional techniques are usually needed to achieve satisfactory long-term results or to solve unexpected intraoperative events.

Grafting of a bifurcation lesion has usually the advantage of supplying both limbs of the bifurcation with a single graft; however, the presence of multiple atheramtous plaques usually complicates the surgical procedure and adversely influences long term patency [7].
Our technique has the advantage of achieving complete revascularization by combined endartrectomy of both limbs of the diseased artery and grafting of both branches using single conduit. A single conduit has also the advantage of reducing the amount of trauma to the aorta which is equally diseased in this subset of patients without adding extra bypass time.

This Technique tries to find a solution for these complicated atheramatous bifurcation lesion and tries also to find a solution for unexpected intraoperative events which may happen during or after endarterectomy in diffusely diseased medium sized vessels.

There is evidence that coronary bypass surgery for small sized vessels has relatively poor outcome [8], however, increasing the size of the vessel by performing a long and a wide anastomosis may improve the flow to these vessels as proved by flow meter study, and postoperative coronary angiography.

In Conclusion, bypass grafting of bifurcation lesion is possible even after endarteresctomy. Our technique is simple, easy to be performed and reproducible. It helps in achieving complete revascularization without adding extra bypass time.

\section{References}

1. Fergson TB, Hammill BG, De Long ER, Grover FL, et al. (2002) A decade changerisk profiles and outcomes for isolated coronary artery bypass grafting procedures, 1990-1998: a report from the STS National Database committee and the Duke clinical research Institute. Ann Thorac surg 73:480-490. [Crossref]

2. Brenowitz JB, Kayser KL, Johnson WD (1988) Results of coronary artery endarterectomy and reconstruction. J Thorac Cardiovasc Surg 95: 1-10. [Crossref]

3. Sundt TM, Camillo CJ, Mendeloff EN, Barner HB, Gay WA Jr (1999) Reappraisal of coronary endarterectomy for the treatment of diffuse coronary artery disease. Ann Thorac Surg 68: 1272-1277. [Crossref]

4. Ferrerira AC, peter AA, Salerno TA, Bolooki H, de Marchena E (2003) Clinica impact of drug-eluting stents in changing referral practices of coronary surgical revascularization in a tertiary care center. Ann Thorac Surg 75: 485-489. [Crossref]

5. Minale C, Nikol S, Zender M, Uebis R, Effect S, et al. (1989) Controversial aspects of coronary endarterectomy. Ann Thorac Surg 48: 235-241. [Crossref]

6. Sirivella S, Gielchinsky I, parsonnet V (2005) Results of coronary artery endarterectomy and coronary artery bypass grafting for diffuse coronary artery disease. Ann Thorac Surg 80:1738-1744. [Crossref]

7. Byrne JG, Karavas AN, Gudbjartson T, Leacche M, Rawn JD, et al. (2004) Left anterior descending coronary endarterectomy: early and late results in 196 consecutive patients. Ann Thorac Surg 78: 867-873. [Crossref]

8. O' Connor NJ, Morton JR, Bikmeyer JD, Olmstead EM, O' Conner GT (1996) Effect of coronary surgery artery diameter in patients undergoing coronary bypass surgery. Circulation 93: 652-655. [Crossref]

Copyright: (C2018 Sayed S. This is an open-access article distributed under the terms of the Creative Commons Attribution License, which permits unrestricted use, distribution, and reproduction in any medium, provided the original author and source are credited. 